\title{
Geriatric Hip Fractures - The Need for Co-Managed Care
}

\section{Gregory Gebauer*}

Department of Orthopedic Spine Surgeon, Advanced Orthopedic Center, Port Charlotte, Florida, USA

Osteoporotic fragility fractures are a significant cause of morbidity for the geriatric population. One third of women and one fifth of men over 50 will sustain a fragility fracture in their lifetime [1]. In the United States alone, this adds up to an estimated 2 million fractures each year [1]. These numbers are expected to rise in future years as the baby boomer generation advances in age. Conservative estimates have the number of osteoporotic hip fractures increasing from 330,000 yearly (at present) to 550,000 by $2040[2,3]$

Of ostoeoporotic fractures, hip fractures are commonly believed to have the highest morbidity and mortality. Less than half of these patients will regain the same level of pre-injury function and a significant number will lose their independence. These fractures also represent a significant burden on the health care system. In 2007 the cost of hip fracture care was estimated at $\$ 17$ billion and it is expected to grow to $\$ 62$ billion by 2040 [4]

The acute and post-injury management of patients with hip and other fragility fractures represent a unique medical challenge. Many of these patients will have complex acute and chronic medical conditions best treated and managed by internists and geriatricians. While these physicians are experts in the medical condition of the patient, they often do not understand the orthopedic and fracture specific issues. Conversely, orthopedic surgeons are frequently uncomfortable managing the complex medical conditions that these patients often have. Anesthesiologists, physiatrists and rehabilitation specialists, physical, occupational and speech therapists all have important roles to play in care of these patients. The multiple factors at play and the lack of interdisciplinary understanding among these specialists can lead to miscommunication, delays and gaps in patient care.

In the United States, several hip fracture programs have recently been developed to help bridge the communication gap and develop a comprehensive team approach to the treatment of these patients. These programs involve the orthopedic specialist, internist, and anesthesiologist acutely. The therapist and rehabilitation specialists follow after the acute care has been addressed. Acutely, these programs are designed to minimize time to surgery, promote early ambulation and prevent complications. Long-term, these programs can help to prevent future fractures by assessing fall risk and treating osteoporosis. Recent experience at the University of Rochester has shown significant decreases in complications $(30.3 \%$ vs. $46.3 \%)$, infections $(2.3 \%$ vs. $19.8 \%$ ) and hospital length of stay (4.6 vs 8.3 days) [5-7]. Additionally patients were noted to have shorter times to surgery, fewer cases thromboembolism, and less delirium. These improvements in care have also resulted in lower reoperation and readmission rates as well as significant reduction in healthcare costs.

These programs are growing in number. Several have been adopted internationally, but are still relatively uncommon outside of North America. The AO North America, a non-profit orthopedic education and research group, has developed an educational course to help teach the principles of co-managed care and expand these programs. While the medical community is becoming more aware of the benefits of these programs, there is still much work to be done to expand these programs into the community and provide better care for our patients.

\section{References}

1. Burge R, Dawson-Hughes B, Solomon DH, Wong JB, King A, et al. (2007) Incidence and economic burden of osteoporosis-related fractures in the United States, 2005-2025. J Bone Miner Res 22: 465-475.

2. http://www.hcup-us.ahrq.gov/db/nation/nis/reports/2005niscomparisonrpt.jsp

3. Braithwaite RS, Col NF, Wong JB (2003) Estimating hip fracture morbidity mortality and costs. J Am Geriatr Soc 51: 364-370.

4. Youm T, Koval KJ, Zuckerman JD (1999) The economic impact of geriatric hip fractures. Am J Orthop (Belle Mead NJ) 28: 423-428.

5. Kates SL, Mendelson DA, Friedman SM (2010) Co-managed care for fragility hip fractures (Rochester model). Osteoporos Int 21: S621-S625.

6. Kates SL, Mendelson DA, Friedman SM (2011) The value of an organized fracture program for the elderly: early results. J Orthop Trauma 25: 233-237.

7. Friedman SM, Mendelson DA, Bingham KW, Kates SL (2009) Impact of a comanaged Geriatric Fracture Center on short-term hip fracture outcomes. Arch Intern Med 169: 1712-1717.
*Corresponding author: Gregory Gebauer, Orthopedic Spine Surgeon, Advanced Orthopedic Center, 1641 Tamiami Trail, Suite 1, Port Charlotte, Florida 33948, USA, Tel: 941-629-6262; E-mail: ggebaue1@gmail.com

Received May 23, 2012; Accepted May 23, 2012; Published May 25, 2012

Citation: Gebauer G (2012) Geriatric Hip Fractures - The Need for Co-Managed Care. J Trauma Treat 1:e104. doi:10.4172/2167-1222.1000e104

Copyright: (c) 2012 Gebauer G. This is an open-access article distributed unde the terms of the Creative Commons Attribution License, which permits unrestricted use, distribution, and reproduction in any medium, provided the original author and source are credited. 